\title{
AGN Jet Kinematics on Parsec-Scales: The MOJAVE Program
}

\author{
Matthew Lister and the MOJAVE Collaboration \\ Department of Physics and Astronomy, Purdue University, 525 Northwestern Avenue, West Lafayette, \\ IN 47907, USA; mlister@purdue.edu
}

Academic Editors: Jose L. Gómez, Alan P. Marscher and Svetlana G. Jorstad

Received: 20 July 2016; Accepted: 2 September 2016; Published: 9 September 2016

\begin{abstract}
Very long baseline interferometry offers the best means of investigating the complex dynamics of relativistic jets powered by active galactic nuclei, via multi-epoch, sub-milliarcsecond, full-polarization imaging at radio wavelengths. Although targeted studies have yielded important information on the structures of individual AGN jets, the strong selection effects associated with relativistically beaming imply that general aspects of the flows can only be determined via large statistical studies. In this review I discuss major results from the Monitoring of Jets in Active Galactic Nuclei With VLBA Experiments (MOJAVE) program, which has gathered multi-epoch Very Long Baseline Array (VLBA) data at $15 \mathrm{GHz}$ on over 400 AGN jets over the course of two decades. The sample is large enough to encompass a range of AGN optical class, radio luminosity and synchrotron peak frequency, and has been used to show that within a particular jet, individual bright features have a spread of apparent speed and velocity vector position angle about a characteristic value. We have found that in some cases there is a secular evolution of launch angle direction over time, indicative of evolving narrow energized channels within a wider outflow. The majority of the jet features are superluminal and accelerating, with changes in speed more common than changes in direction. Within approximately 100 pc of the AGN, the flows are generally accelerating, while beyond this distance the flows begin to decelerate or remain nearly constant in speed. We also find evidence for a maximum bulk flow Lorentz factor of 50 in the pc-scale radio regime, and a trend of higher jet speeds in lower-synchrotron peaked and gamma-ray-loud blazars.
\end{abstract}

Keywords: BL Lacertae objects: general; galaxies: active; galaxies: jets; quasars: general; radio continuum: galaxies

\section{Introduction}

The techniques of radio-wavelength Very Long Baseline Interferometry (VLBI) make it possible to study the the time evolution of AGN jets on parsec-scales, despite their enormous cosmological distances. The advent of the first dedicated VLBI array: the Very Long Baseline Array (VLBA) in 1994 represented a major advance for jet kinematics studies, by making it possible to observe relatively large samples of jets at regular intervals. In this review, I will focus on one of the largest and well-known AGN jet VLBI studies, the $2 \mathrm{~cm}$ Survey [1], and its successor, the MOJAVE (Monitoring of Jets in Active Galactic Nuclei With VLBA Experiments) program [2].

MOJAVE is distinct in terms of its large sample size (several hundred AGN, distributed across the northern $2 / 3$ of the celestial sphere), its long continuous time baselines, and its full polarization observations. Other past and current AGN jet monitoring programs are complementary to MOJAVE with regards to their sky coverage (southern hemisphere: TANAMI [3]), observing frequency (43 GHz: Boston U. program [4]; 8 GHz: USNO RFID [5]; 5 GHz: CJ-F survey [6]) and monitoring of faint TeV blazar jets [7]. 


\section{Program Description}

The MOJAVE program, together with the $2 \mathrm{~cm}$ VLBA survey, are the longest continuous programs on the VLBA, dating back to the array's inauguration in 1994. The survey's initial goals were to obtain total intensity-only, milliarcsecond-scale resolution images of $\sim 200$ bright and compact AGN jets at regular intervals to study their kinematics [8]. In 2002, the survey was streamlined to focus on full polarization $15 \mathrm{GHz}$ VLBA observations of a complete sample of 135 northern AGN [9], which was later extended to encompass every non-lensed AGN above declination $-30^{\circ}$ that exceeded $1.5 \mathrm{Jy}$ in $15 \mathrm{GHz}$ VLBA flux density between 1994.0 and 2010.0 [10]. In 2006, we further expanded our observations to encompass additional compact radio-loud AGN in anticipation of the Fermi gamma-ray space telescope mission [11]. These included a complete Fermi $\gamma$-ray flux-limited sample (the 1 FM; [12]), and a low radio luminosity sample designed to probe the jet kinematics of less highly-beamed AGN [13].

In 2013, we took advantage of a major VLBA sensitivity upgrade to focus on a set of weaker radio AGN associated with hard-spectrum, high synchrotron peaked (HSP), $\gamma$-ray loud AGN. These are predominantly BL Lac objects, and dominate the Fermi AGN catalog at lower $\gamma$-ray flux levels. We have recently been granted twelve $24 \mathrm{~h}$ VLBA sessions per year through August 2019 to observe 41 AGN from our previous samples that display strongly accelerating jet features, as well as 57 new target AGN from the Fermi LAT-monitored list (http://fermi.gsfc.nasa.gov/ssc/data/access/lat/msl_lc/), the 2FHL and 3FGL Fermi catalogs [14,15], and the RoboPol sample [16]. With these new additions the total number of AGN jets that have been monitored by MOJAVE will exceed 500. In 2017-19 we will also be obtaining a limited set of VLBA epochs at $43 \mathrm{GHz}$ on 14 highly-compact AGN jets whose speeds could not be measured at $15 \mathrm{GHz}$.

The MOJAVE VLBA observations are carried out in 24 hour-long segments at monthly intervals, and in each segment a small subset of the total AGN sample is observed. The subset is chosen on the basis of their individual rates of angular jet motion, with several very fast ( $>2$ mas $\cdot$ year $^{-1}$ ) jets being observed once per month, while the slowest jets are observed only once every few years. When new jets are added to the program, several initially closely spaced epochs are obtained in order to establish their optimal monitoring cadence.

Through collaborative efforts, we have also obtained a substantial amount of supporting observations over the last decade using the Jansky VLA, LOFAR, HST, Chandra, Swift, and VERITAS telescopes, as well as several ground-based optical facilities. Our VLBA flux density data are complemented in the radio by the long term, frequently-sampled radio variability data sets from the UMRAO, F-GAMMA, RATAN-600, and OVRO single-dish monitoring programs. In parallel VLA observations completed in summer 2015, we have obtained deep multi-configuration L and C-band images of our complete $1.5 \mathrm{Jy}$ and 1FM samples, to follow up our earlier study of AGN jet power and morphology on kiloparsec scales [17].

\section{Research Highlights}

A primary goal of the MOJAVE program is to investigate the pc-scale jet kinematics of complete samples of AGN, and how they are related to other properties, including gamma-ray activity, intraday variability, and overall jet power. Our kinematic analysis is carried out on individual bright jet features (modeled as Gaussians in the interferometric visibility plane) using the bright, optically thick feature located near the base of the jet (the "core") as a fiducial stationary point. The rms sky position accuracy is typically $0.05-0.1$ mas, and fits to the sky trajectories are made separately in the R.A. and declination directions (Figure 1). For the jets with sufficient data, we also fit accelerating trajectories. The limited angular resolution of the VLBA at $15 \mathrm{GHz}$ ( 0.5 mas) limits the distance at which any new feature can begin to be tracked, and due to the exponential fall-off in synchrotron radio intensity down the jet, most can only be tracked out to 10 mas from the core. Given the typical redshifts and viewing angles ( $<10$ deg.) of these AGN jets, we are therefore probing jet kinematics at de-projected distances $10-1000 \mathrm{pc}$ from the central engine. 


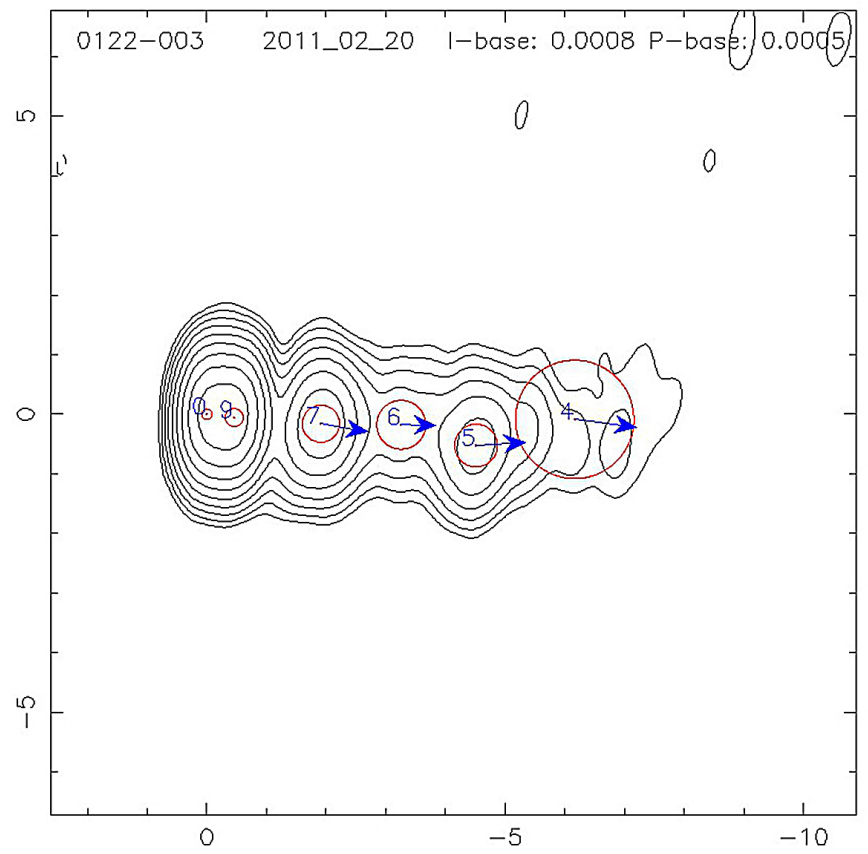

Figure 1. Monitoring of Jets in Active Galactic Nuclei With VLBA Experiments (MOJAVE) 15 GHz contour map of the jet of PKS 0122-003 in February 2011, with fitted circular Gaussian jet features and vector arrows showing the direction of their motion on the sky. The lengths of the arrows are proportional to the proper motions, which range from 120 to 230 mas $\cdot$ year $^{-1}$ (7-12 c).

\subsection{Jet Kinematics}

Our most recent kinematics analyses $[18,19]$ have shown that within an individual pc-scale AGN jet, the bright features typically have a range of speeds, clustered around a characteristic median value. Stationary/slow pattern speed features are often present ( $6 \%$ of all studied features) and may be associated with recollimation shocks in the flow. These are more prevalent in weak-lined BL Lac jets $(25 \%)$ than quasars $(15 \%)$, and the majority are located within 1 mas of the core feature.

The distribution of maximum jet speeds seen in each jet is peaked at low values, and tapers off sharply at high speeds (Figure 2). This is indicative of a parent population with a distribution of bulk flow Lorentz factors ranging up to $\Gamma \simeq 50$. The distribution in Figure 2 is similar to the early results from the CJ-F survey, which indicated a parent power law Lorentz factor distribution of $p(\Gamma) \propto \Gamma^{-1.5}$ [20]. A detailed analysis of the complete MOJAVE $1.5 \mathrm{Jy}$ sample jet speeds is currently underway.

We also have found that the majority of AGN jet features undergo significant accelerations, and that changes in speed are larger than changes in direction [18]. Over $80 \%$ of the jets we have studied show at least one feature that is either accelerating or has a velocity vector that does not point back towards the core. Unlike protostellar and X-ray binary jets, which show exclusively ballistic behavior [21], the accelerations in AGN illustrate the strong influence of MHD processes on the flow, as we recently explored in a detailed study of MHD waves in the jet of the nearby AGN BL Lacertae [22]. Within approximately 100 pc of the base of the jet, features tend to show increasing rather than decreasing apparent speed. This suggests that the flows are still being organized on pc-scales, a favored possible site for high energy photon production. Further downstream, we have witnessed sudden changes in moving feature trajectories [23] that are likely associated with large-scale collimation. 


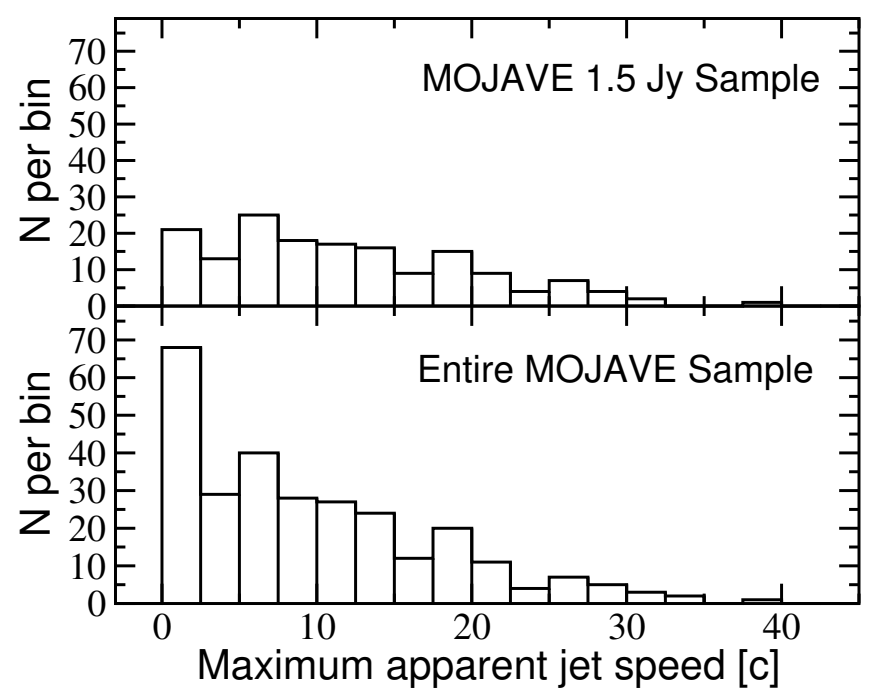

Figure 2. Top panel: distribution of maximum observed jet speed for AGN in the flux-density limited MOJAVE 1.5 Jy sample. Lower panel: maximum speed distribution for all jets measured to date by the MOJAVE program.

\subsection{Swinging Jets}

Our program has also established that the apparent direction of the pc-scale flow on the sky can frequently undergo significant changes (typically $1-3 \mathrm{deg} \cdot \mathrm{year}^{-1}$, and ranging up to $10 \mathrm{deg} \cdot \mathrm{year}^{-1}$ ) on decadal timescales [13]. Many AGN jets show a gradual swing in the projected sky position angle at which successive features are seen to emerge from the VLBI core region (Figure 3). In some cases the swings in position angle show hints of periodicity, which has been suggested as evidence for a binary black hole system in which the companion is providing an external torque on the accretion disk, thereby causing the jet axis to precess [24]. Indeed, many AGN jets show "smoking-gun" evidence of long-term precession on kpc scales $[25,26]$. The situation is likely complicated however, since many of these new features accelerate after leaving the immediate region of the core.
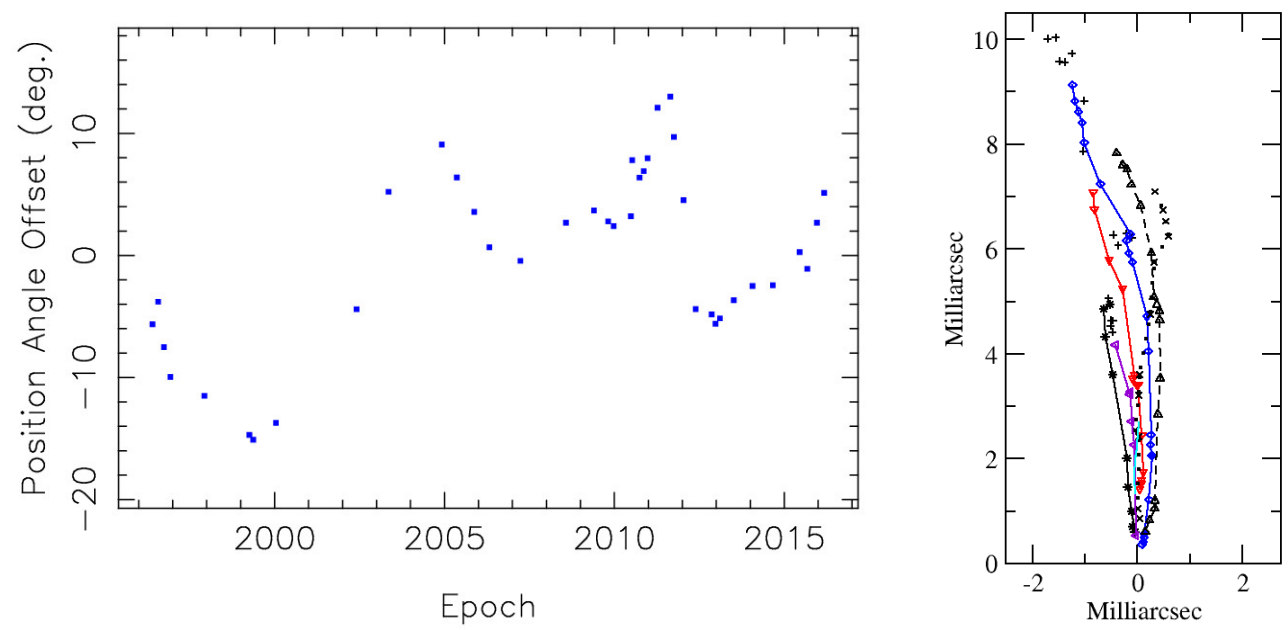

Figure 3. Left: Plot of inner jet position angle versus time for the quasar $1222+216$, based on MOJAVE Very Long Baseline Array (VLBA) data; Right: Sky trajectories of moving features in this jet over a 15 year period. 
When MOJAVE images taken over 15-20 years are stacked, a smooth, conical jet shape is frequently revealed, even when at individual epochs the jet can appear quite bent and knotty [27]. This indicates that in most jets only a portion of a much wider flow is strongly energized at any given time. Energized "ribbons" in the flow are marked by bright, synchrotron-emitting knots, which move at apparent superluminal speed downstream. Given the energetics and enormous torques involved, it is more likely that these ribbons, and not the whole jet, are precessing. Blazar jets that are precessing will likely experience large changes in Doppler factor which should be reflected in their apparent jet speeds, accelerations, and variability timescales in the radio and $\gamma$-ray regimes.

\subsection{Trends with Synchrotron Peak Location}

The MOJAVE program has also found connections between the pc-scale radio jet properties and the overall spectral energy distributions (SED) of blazars. The latter consists of two broad peaks, each spanning several orders of magnitude in photon energy. The first peak arises from synchrotron emission of relativistic electrons in the jet plasma, while the second is widely believed to be due to inverse Compton scattering of ambient photons or high energy particle cascades. Within the known blazar population, there is a large range (at least 4 dex) of synchrotron peak frequency, from the IR to soft X-rays. Since nearly all blazar surveys prior to Fermi were constructed using relatively narrow energy pass bands, each flux-limited survey would preferentially select different types of blazars, based on the pass band location with respect to their SED peak(s). Fermi's LAT instrument has good sensitivity over the broad range of $0.1 \mathrm{MeV}$ to a few hundred $\mathrm{GeV}$, and is thus capable of detecting blazars with a wide range of SED peak frequencies. Furthermore, at $\gamma$-ray energies there is little obscuration or spectral contamination from the host galaxy, and the typically weak $\gamma$-ray emission from jets on kiloparsec scales means that AGN can be selected purely on the basis of relativistically beamed jet emission.

In 2009, we carried out a joint MOJAVE-LAT team study of the brightest $\gamma$-ray as well as radio-selected AGN in the northern sky [12]. The latter consisted of the complete 1.5 Jy MOJAVE sample, while the former (the 1FM sample) is comprised all AGN above dec $-30^{\circ}$ and $|b|>10^{\circ}$, with $>0.1 \mathrm{GeV}$ median energy flux $\geq 3 \times 10^{-11} \mathrm{MeV} \cdot \mathrm{cm}^{-2} \cdot \mathrm{s}^{-1}$. We found that for the (weak lined) BL Lacs, there is a tight correlation between synchrotron peak frequency and the $\gamma$-ray-to-radio luminosity ratio. No such trend exists for (broad lined) quasars. We argued that in order to have such a good correlation, there must be a narrow range of SED shape, implying that the only major distinction between bright HSP and LSP BL Lacs is the peak energy of their relativistic electron population. The trend also implies a similar amount of Doppler beaming in the $\gamma$-ray and radio regimes, which suggests that in BL Lacs, the dominant $\gamma$-ray mechanism is inverse Compton scattering of synchrotron photons originating from the jet itself, and not an external ambient photon field.

Our findings are difficult to reconcile with the popular paradigm for HSP BL Lacs such as Mrk 421 and PKS 2155-304, which are detected at TeV energies by Cherenkov air shower telescopes. In order to explain their extremely rapid $\mathrm{TeV}$ flux variations, very high bulk flow Doppler factors must be invoked; otherwise the $\gamma$-rays would not be able to escape the (putatively small) emitting region due to catastrophic pair-creation losses [28]. However, multi-epoch VLBI studies of a small number of TeV-emitting jets by [29], as well as our own measurements [13], show slow speeds and little structural variability in their pc-scale radio jets (Figure 4). This has led to a popular interpretation in which the $\mathrm{TeV} \gamma$-ray rays originate in a high-Lorentz factor spine, while the radio emission comes from a much slower surrounding sheath [30]. The main difficulties with this model are twofold. First, our 1FM study showed no major differences in the parent populations of $\gamma$-ray and radio-selected blazars, and strong correlations between the $\gamma$-ray and radio jet emission, which are hard to explain if these respective emission regions are located in different regions of the jet, with substantially different Doppler beaming factors. Second, the trends we found for the BL Lac objects are continuous over the entire range of synchrotron peak frequency, suggesting that they are all from the same parent population. In other words, spine-sheath structures, if they exist, should be present in both high (HSP) 
and low-synchrotron-peaked (LSP) BL Lacs. Our MOJAVE AGN speed measurements indicate that jet Doppler factors range up to 100 [19], which would also imply that HSP BL Lacs should be among the most highly aligned and boosted jets in the population, according to the spine-sheath model.

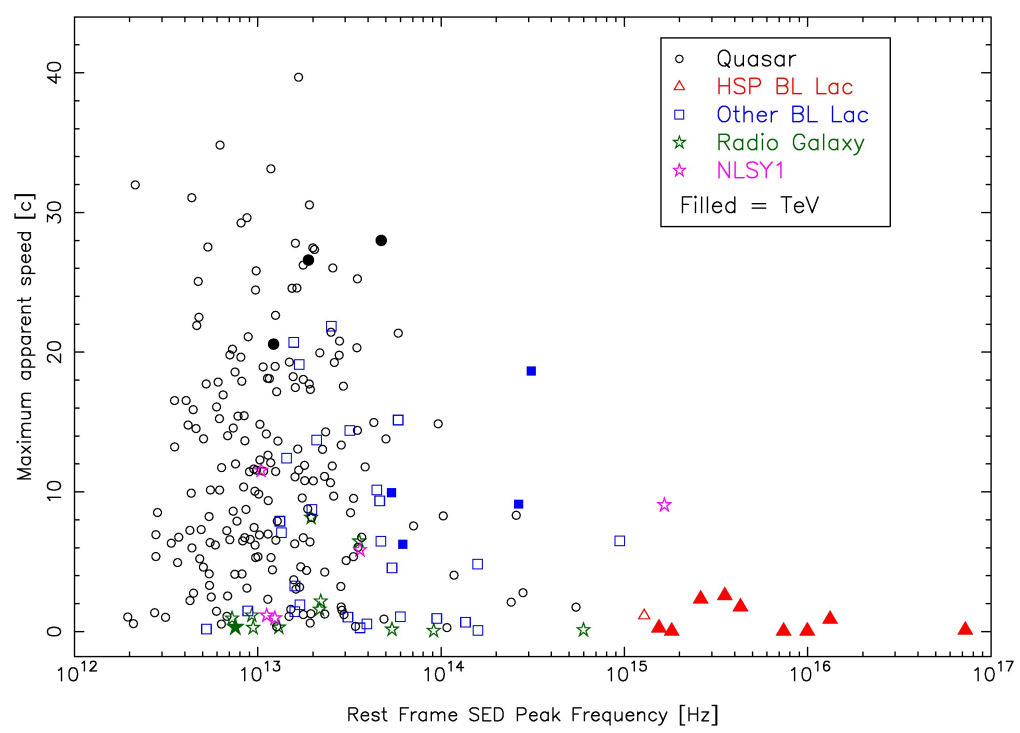

Figure 4. Plot of maximum apparent jet speed vs. synchrotron spectral energy distributions (SED) peak frequency for jets measured by the MOJAVE program.

There has been little need, however, to invoke spine-sheath structures in the LSP BL Lacs, as very few have been detected at TeV energies, and they display superluminal speeds and high bulk Lorentz factors in the radio regime [31]. A study of a major TeV flare in BL Lacertae by [32] showed that both its high energy flux variability and its overall SED could be explained using a Doppler factor consistent with its measured pc-scale jet speed. Several alternatives to the spine-sheath model have been proposed, including individually misaligned high-speed regions within the jet (e.g., [33]), decelerating flows [34] and magnetically-driven non-stationary jet acceleration [35]. The MOJAVE program has provided observational support for these models, with our finding that AGN $\gamma$-ray emission is non-isotropic in the jet rest frame [36].

We are currently gathering speed information for a larger number of ISP and HSP blazars to investigate these issues, as well as whether the kinematic acceleration properties of low and high synchrotron peaked jets differ, as predicted by magnetized outflow models (e.g., [35]). Our preliminary data indicate that their radio core compactness, fractional polarization level, and flux variability are considerably lower than in flat spectrum quasars, suggesting that high synchrotron peaked jets are in fact only moderately Doppler boosted. The apparent speeds of their jet features appear to be generally slow (Figure 4), in agreement with other VLBI surveys [29].

\subsection{Narrow-Line Seyfert I Jets}

Although the Fermi AGN catalog is heavily dominated by flat spectrum radio quasars and BL Lac objects, a third class of gamma-ray AGN has recently identified [37]. These narrow-line Seyfert I galaxies (NLSY1) are known to have relatively small black-hole masses $\left(10^{6}-10^{8} \mathrm{M}_{\odot}\right)$ and likely high Eddington ratios. To date we have studied the kinematics of five NLSY1 AGN in MOJAVE, and find three to have significant superluminal jet motion [19]. This implies that they share many similar characteristics to typical blazar jets, i.e., low viewing angle and high bulk Lorentz factor. Their jets are typically less luminous than flat spectrum quasars, and more similar to those of nearby BL Lac objects (Figure 5). 


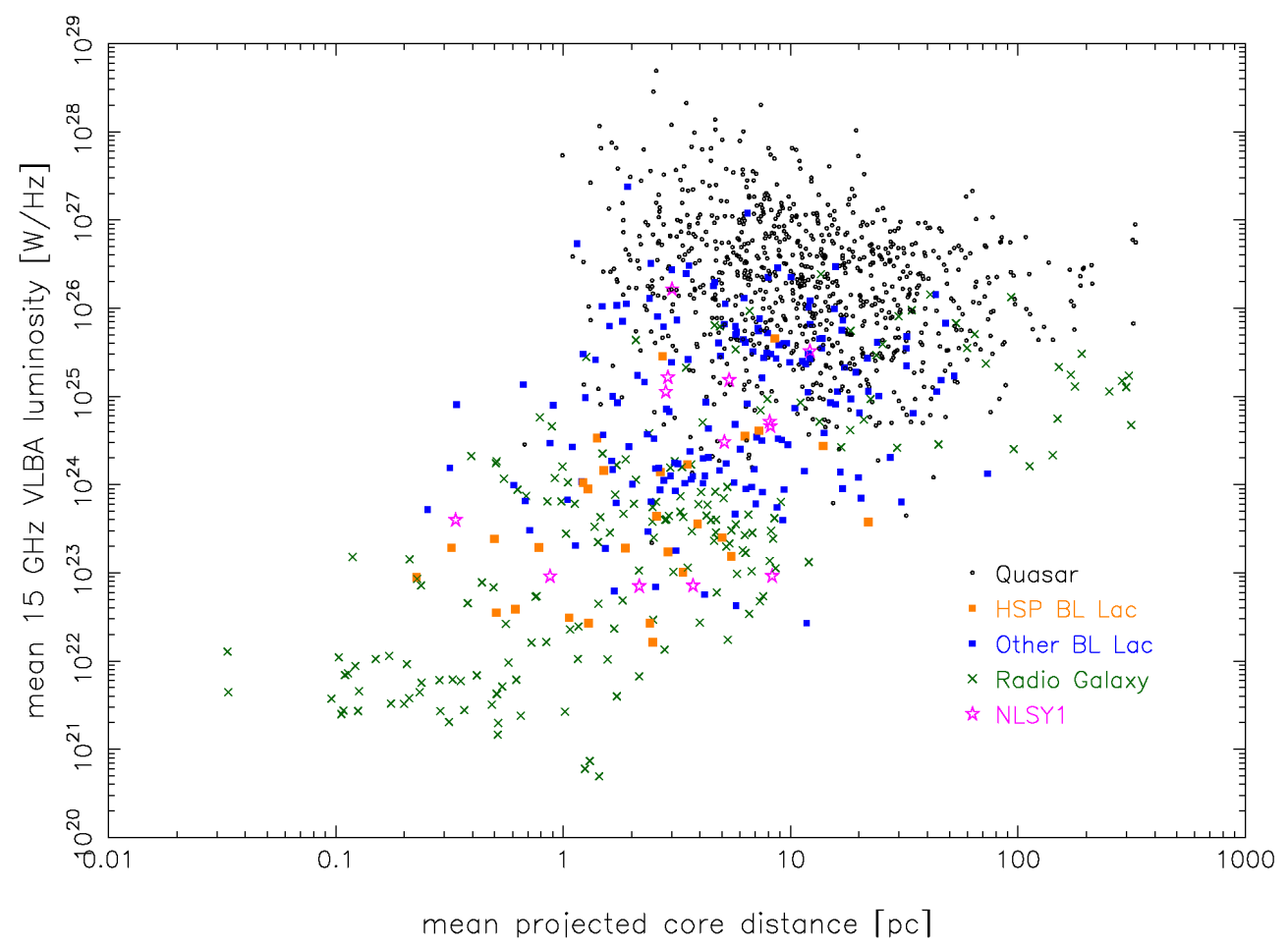

Figure 5. Plot of $15 \mathrm{GHz}$ luminosity versus projected distance downstream from the core feature, for jet features in the MOJAVE survey. Black circles represent quasars, orange squares high spectral peaked BL Lacs, blue squares other BL Lacs, green crosses radio galaxies, and purple stars NLSY1 galaxies.

\section{Summary}

The MOJAVE program has demonstrated the vast capabilities of the VLBA for investigating the highly relativistic outflows powered by supermassive black holes in AGN. Future planned VLBA sensitivity upgrades will enhance these capabilities even further, allowing us to probe the complex time-evolving nature of a wider range of fainter jets associated with low-luminosity and hard spectrum gamma-ray AGN. MOJAVE continues to provide a large amount of fully reduced data to the community via its website (http:/ / www.astro.purdue.edu/MOJAVE), as well as time-lapse movies and educational materials.

Acknowledgments: The MOJAVE project was supported by NASA-Fermi GI grant NNX15AU76G. The National Radio Astronomy Observatory is a facility of the National Science Foundation operated under cooperative agreement by Associated Universities, Inc. This work made use of the Swinburne University of Technology software correlator [38], developed as part of the Australian Major National Research Facilities Programme and operated under licence.

Conflicts of Interest: The authors declare no conflict of interest.

\section{References}

1. Kellermann, K.I.; Vermeulen, R.C.; Zensus, J.A.; Cohen, M.H. Sub-Milliarcsecond Imaging of Quasars and Active Galactic Nuclei. Astron. J. 1998, 115, 1295-1318.

2. $\quad$ Lister, M.L.; Aller, H.D.; Aller, M.F.; Cohen, M.H.; Homan, D.C.; Kadler, M.; Kellermann, K.I.; Kovalev, Y.Y.; Ros, E.; Savolainen, T.; et al. MOJAVE: Monitoring of Jets in Active Galactic Nuclei with VLBA Experiments. V. Multi-Epoch VLBA Images. Astron. J. 2009, 137, 3718-3729.

3. Ojha, R.; Kadler, M.; Böck, M.; Booth, R.; Dutka, M.S.; Edwards, P.G.; Fey, A.L.; Fuhrmann, L.; Gaume, R.A.; Hase, H.; et al. TANAMI: Tacking active galactic nuclei with austral milliarcsecond interferometry. I. First-epoch $8.4 \mathrm{GHz}$ images. Astron. Astrophys. 2010, 519, A45.

4. Jorstad, S.G.; Marscher, A.P.; Agudo, I.; Smith, P.S.; Larionov, V.M.; Lähteenmäki, A. Multi-Frequency Observations of Gamma-Ray Blazar 1633+382. J. Astrophys. Astron. 2011, 32, 239-242. 
5. Piner, B.G.; Pushkarev, A.B.; Kovalev, Y.Y.; Marvin, C.J.; Arenson, J.G.; Charlot, P.; Fey, A.L.; Collioud, A.; Voitsik, P.A. Relativistic Jets in the Radio Reference Frame Image Database. II. Blazar Jet Accelerations from the First 10 Years of Data (1994-2003). Astrophys. J. 2012, 758, 84.

6. Britzen, S.; Vermeulen, R.C.; Taylor, G.B.; Campbell, R.M.; Pearson, T.J.; Readhead, A.C.S.; Xu, W.; Browne, I.W.A.; Henstock, D.R.; Wilkinson, P. A multi-epoch VLBI survey of the kinematics of CJF sources. I. Model-fit parameters and maps. Astron. Astrophys. 2007, 472, 763-771.

7. Piner, B.G.; Edwards, P.G. First-epoch VLBA Imaging of $20 \mathrm{New} \mathrm{TeV} \mathrm{Blazars.} \mathrm{Astrophys.} \mathrm{J.} \mathrm{2014,} 797,25$.

8. Kellermann, K.I.; Lister, M.L.; Homan, D.C.; Vermeulen, R.C.; Cohen, M.H.; Ros, E.; Kadler, M.; Zensus, J.A.; Kovalev, Y.Y. Sub-Milliarcsecond Imaging of Quasars and Active Galactic Nuclei. III. Kinematics of Parsec-scale Radio Jets. Astrophys. J. 2004, 609, 539-563.

9. Lister, M.L.; Homan, D.C. MOJAVE: Monitoring of Jets in Active Galactic Nuclei with VLBA Experiments. I. First-Epoch 15 GHz Linear Polarization Images. Astron. J. 2005, 130, 1389-1417.

10. Lister, M.L.; Aller, M.F.; Aller, H.D.; Hovatta, T.; Max-Moerbeck, W.; Readhead, A.C.S.; Richards, J.L.; Ros, E. Why Have Many of the Brightest Radio-loud Blazars Not Been Detected in Gamma-Rays by Fermi? Astrophys. J. Lett. 2015, 810, L9.

11. Atwood, W.B.; Abdo, A.A.; Ackermann, M.; Althouse, W.; Anderson, B.; Axelsson, M.; Baldini, L.; Ballet, J.; Band, D.L.; Barbiellini, G.; et al. The Large Area Telescope on the Fermi Gamma-Ray Space Telescope Mission. Astrophys. J. 2009, 697, 1071-1102.

12. Lister, M.L.; Aller, M.; Aller, H.; Hovatta, T.; Kellermann, K.I.; Kovalev, Y.Y.; Meyer, E.T.; Pushkarev, A.B.; Ros, E.; MOJAVE Collaboration; et al. $\gamma$-Ray and Parsec-scale Jet Properties of a Complete Sample of Blazars From the MOJAVE Program. Astrophys. J. 2011, 742, 27.

13. Lister, M.L.; Aller, M.F.; Aller, H.D.; Homan, D.C.; Kellermann, K.I.; Kovalev, Y.Y.; Pushkarev, A.B.; Richards, J.L.; Ros, E.; Savolainen, T. MOJAVE. X. Parsec-scale Jet Orientation Variations and Superluminal Motion in Active Galactic Nuclei. Astron. J. 2013, 146, 120.

14. Nolan, P.L.; Abdo, A.A.; Ackermann, M.; Ajello, M.; Allafort, A.; Antolini, E.; Atwood, W.B.; Axelsson, M.; Baldini, L.; Ballet, J.; et al. Fermi Large Area Telescope Second Source Catalog. Astrophys. J. Suppl. 2012, doi:10.1088/0067-0049/199/2/31.

15. Acero, F.; Ackermann, M.; Ajello, M.; Albert, A.; Atwood, W.B.; Axelsson, M.; Baldini, L.; Ballet, J.; Barbiellini, G.; Bastieri, D.; et al. Fermi Large Area Telescope Third Source Catalog. Astrophys. J. Suppl. 2015, 218, 23.

16. Pavlidou, V.; Angelakis, E.; Myserlis, I.; Blinov, D.; King, O.G.; Papadakis, I.; Tassis, K.; Hovatta, T.; Pazderska, B.; Paleologou, E.; et al. The RoboPol optical polarization survey of gamma-ray-loud blazars. Mon. Not. R. Astron. Soc. 2014, 442, 1693-1705.

17. Kharb, P.; Lister, M.L.; Cooper, N.J. Extended Radio Emission in MOJAVE Blazars: Challenges to Unification. Astrophys. J. 2010, 710, 764-782.

18. Homan, D.C.; Lister, M.L.; Kovalev, Y.Y.; Pushkarev, A.B.; Savolainen, T.; Kellermann, K.I.; Richards, J.L.; Ros, E. MOJAVE. XII. Acceleration and Collimation of Blazar Jets on Parsec Scales. Astrophys. J. 2015, 798, 134.

19. Lister, M.L.; Aller, M.F.; Aller, H.D.; Homan, D.C.; Kellermann, K.I.; Kovalev, Y.Y.; Pushkarev, A.B.; Richards, J.L.; Ros, E.; Savolainen, T. MOJAVE: XIII. Parsec-scale AGN Jet Kinematics Analysis Based on 19 years of VLBA Observations at $15 \mathrm{GHz}$. Astron. J. 2016, 152, 12.

20. Lister, M.L.; Marscher, A.P. Statistical Effects of Doppler Beaming and Malmquist Bias on Flux-limited Samples of Compact Radio Sources. Astrophys. J. 1997, 476, 572-588.

21. Miller-Jones, J.C.A. High-resolution radio observations of X-ray binaries. J. Phys. Conf. Ser. 2008, 131, 012057.

22. Cohen, M.H.; Meier, D.L.; Arshakian, T.G.; Clausen-Brown, E.; Homan, D.C.; Hovatta, T.; Kovalev, Y.Y.; Lister, M.L.; Pushkarev, A.B.; Richards, J.L.; et al. Studies of the Jet in BL Lacertae. II. Superluminal Alfvén Waves. Astrophys. J. 2015, 803, 3.

23. Homan, D.C.; Lister, M.L.; Kellermann, K.I.; Cohen, M.H.; Ros, E.; Zensus, J.A.; Kadler, M.; Vermeulen, R.C. Jet Collimation in Action: Realignment on Kiloparsec Scales in 3C 279. Astrophys. J. Lett. 2003, 589, L9-L12.

24. Caproni, A.; Abraham, Z.; Monteiro, H. Parsec-scale jet precession in BL Lacertae (2200+420). Mon. Not. R. Astron. Soc. 2013, 428, 280-290.

25. Alexander, P. A radio study of 3C 390.3-A precessing jet? Mon. Not. R. Astron. Soc. 1985, 213, 743-752. 
26. Steenbrugge, K.C.; Blundell, K.M. Multiwavelength study of CygnusA-I. Precession and slow jet speeds from radio observations. Mon. Not. R. Astron. Soc. 2008, 388, 1457-1464.

27. Homan, D.C. Physical Properties of Jets in AGN. Int. J. Mod. Phys. Conf. Ser. 2012, 8, 163-171.

28. Aharonian, F.; Akhperjanian, A.G.; Bazer-Bachi, A.R.; Behera, B.; Beilicke, M.; Benbow, W.; Berge, D.; Bernlöhr, K.; Boisson, C.; Bolz, O.; et al. An Exceptional Very High Energy Gamma-Ray Flare of PKS 2155-304. Astrophys. J. Lett. 2007, 664, L71-L74.

29. Piner, B.G.; Pant, N.; Edwards, P.G. The Jets of TeV Blazars at Higher Resolution: $43 \mathrm{GHz}$ and Polarimetric VLBA Observations from 2005 to 2009. Astrophys. J. 2010, 723, 1150-1167.

30. Ghisellini, G.; Tavecchio, F.; Chiaberge, M. Structured jets in TeV BL Lac objects and radiogalaxies. Implications for the observed properties. Astron. Astrophys. 2005, 432, 401-410.

31. Lister, M.L.; Cohen, M.H.; Homan, D.C.; Kadler, M.; Kellermann, K.I.; Kovalev, Y.Y.; Ros, E.; Savolainen, T.; Zensus, J.A. MOJAVE: Monitoring of Jets in Active Galactic Nuclei with VLBA Experiments. VI. Kinematics Analysis of a Complete Sample of Blazar Jets. Astron. J. 2009, 138, 1874-1892.

32. Arlen, T.; Aune, T.; Beilicke, M.; Benbow, W.; Bouvier, A.; Buckley, J.H.; Bugaev, V.; Cesarini, A.; Ciupik, L.; Connolly, M.P.; et al. Rapid TeV Gamma-Ray Flaring of BL Lacertae. Astrophys. J. 2013, 762, 92-105.

33. Giannios, D.; Uzdensky, D.A.; Begelman, M.C. Fast TeV variability from misaligned minijets in the jet of M87. Mon. Not. R. Astron. Soc. 2010, 402, 1649-1656.

34. Georganopoulos, M.; Kazanas, D. Decelerating Flows in TeV Blazars: A Resolution to the BL Lacertae-FR I Unification Problem. Astrophys. J. Lett. 2003, 594, L27-L30.

35. Lyutikov, M.; Lister, M. Resolving Doppler-factor Crisis in Active Galactic Nuclei: Non-steady Magnetized Outflows. Astrophys. J. 2010, 722, 197-203.

36. Savolainen, T.; Homan, D.C.; Hovatta, T.; Kadler, M.; Kovalev, Y.Y.; Lister, M.L.; Ros, E.; Zensus, J.A. Relativistic beaming and gamma-ray brightness of blazars. Astron. Astrophys. 2010, 512, A24.

37. Ackermann, M.; Ajello, M.; Atwood, W.B.; Baldini, L.; Ballet, J.; Barbiellini, G.; Bastieri, D.; Becerra Gonzalez, J.; Bellazzini, R.; Bissaldi, E.; et al. The Third Catalog of Active Galactic Nuclei Detected by the Fermi Large Area Telescope. Astrophys. J. 2015, 810, 14.

38. Deller, A.T.; Brisken, W.F.; Phillips, C.J.; Morgan, J.; Alef, W.; Cappallo, R.; Middelberg, E.; Romney, J.; Rottmann, H.; Tingay, S.J.; et al. DiFX-2: A More Flexible, Efficient, Robust, and Powerful Software Correlator. Publ. Astron. Soc. Pac. 2011, 123, 275-287.

(C) 2016 by the authors; licensee MDPI, Basel, Switzerland. This article is an open access article distributed under the terms and conditions of the Creative Commons Attribution (CC-BY) license (http:/ / creativecommons.org/licenses/by/4.0/). 